\title{
Remissionsinduktion mit Infliximab bei therapierefraktärer Wegenerscher Granulomatose - Verlaufsbeobachtung ${ }^{1}$
}

\section{P. Lamprecht ${ }^{1}$}

O. Arbach ${ }^{1}$

J. Voswinkel ${ }^{1}$

T. Lilienthal ${ }^{1}$

B. Nölle ${ }^{2}$

M. Heller ${ }^{3}$

A. Gause ${ }^{1}$

W. L. Gross ${ }^{1}$

\section{Induction of remission with infliximab in therapy-refractory Wegener's granulomatosis - Follow-up of six patients}

Hintergrund und Fragestellung: Therapierefraktäre Verläufe einer Wegenerschen Granulomatose (WG) unter der Standardtherapie zur Remissionsinduktion (Cyclophosphamid und Steroide) haben eine schlechte Prognose. Wir berichten über die Ergebnisse einer Langzeitbeobachtung von sechs Patienten mit therapierefraktärer WG unter Standardmedikation (Fauci-Schema) und progredienter visusbedrohender retroorbitaler bzw. pulmonaler und renaler Beteiligung. Lassen sich mit dem AntiTNF- $\alpha$-Antikörpers Infliximab langfristige Remissionen erzielen und welche Nebenwirkungen treten auf?

Patienten und Methodik: Die Patienten erhielten Infliximab (zunächst $3 \mathrm{mg} / \mathrm{kg}$ ) zusätzlich zur vorbestehenden Cyclophosphamid- und Steroidtherapie nach dem Fauci-Schema. Die TherapieIntervalle zwischen den ersten beiden Infusionen betrugen 2 Wochen, danach 4 Wochen. Später erhielten die Patienten unter dem Eindruck einer höheren Effektivität $5 \mathrm{mg} / \mathrm{kg}$ Infliximab.

Ergebnisse: Bei fünf Patienten wurde nach 4-6 Infusionen eine Remission erzielt. Vier Patienten sind nach 16-26 Monaten weiterhin in Vollremission. Ein Patient erlitt unter remissionserhaltender Therapie mit Azathioprin nach 12 Monaten ein Rezidiv. Bei einem weiteren Patienten wurde die Infliximab-Therapie aufgrund einer vermuteten systemischen Infektion vorzeitig beendet. Bei beiden Patienten konnte bei pulmonalem Rezidiv bzw. weiterhin progredienter, visusbedrohender Protrusio bulbi, hoher kumulativer Cyclophosphamid-Dosis (100 g bzw. 275 g) und Cyclophosphamid-induzierter hämorrhagischer Zystitis durch erneute Gabe von Infliximab zusätzlich zu Azathioprin eine Remission erzielt werden. Bei einer Patientin wurde nach 12-monatiger remissionserhaltender Therapie ein Bronchus-Karzinoid diagnostiziert.

Folgerungen: Mit dem Anti-TNF- $\alpha$-Antikörper Infliximab eröffnet sich eine neue Therapieoption, um bei einer Patientengruppe mit bisher denkbar schlechter Prognose Remissionen zu erreichen.
Background and question: The prognosis of patients with Wegener's granulomatosis (WG) refractory to standard treatment for the induction of remission (cyclophosphamide and steroids) has been poor so far. We report on the results of the follow-up of six patients refractory to the standard regimen (Fauci's scheme) with progressive, imminent visual loss, pulmonary and renal involvement, respectively. How long can remissions be successfully maintained with anti-TNF- $\alpha$-antibody infliximab? What side effects occur?

Patients and methods: Patients received infliximab $(3 \mathrm{mg} / \mathrm{kg})$ in addition to standard therapy with cyclophosphamide and steroids. Intervals between the first two infliximab infusions were 2 weeks, thereafter 4 weeks. Based on the impression of higher efficacy patients received $5 \mathrm{mg} / \mathrm{kg}$ infliximab for subsequent infusions.

Results: Remission was induced after 4-6 infliximab infusions in five patients. Remission has been maintained in four patients for 16-26 months. After 12 months a pulmonary relapse occurred in one patient, who received azathioprine for the maintenance of remission. Infliximab was stopped in another patient because of a suspected infection. In the light of high cumulative cyclophosphamide doses (100 g/275 g) and cyclophosphamide induced hemorrhagic cystitis, infliximab was added to azathioprine in the two patients with a pulmonary relapse and protrusion of the eye with imminent visual loss, respectively. Remission was induced in both patients. A carcinoid of the bronchus was diagnosed in one patient after 12 months in remission.

Conclusion: Infliximab means a new therapeutic option and offers better perspectives for a patient group with previously bad prognosis.

1 Unterstützt durch Fördermittel seitens der DFG (SFB367/A8: PL, WLG und DFG GA302-3: JV, AG) und Projekt 02.2 des Vereins zur Förderung der Erforschung und Bekämpfung rheumatischer Erkrankungen Bad Bramstedt e.V.(PL).

Institut

${ }^{1}$ Poliklinik für Rheumatologie, Universitätsklinikum Lübeck, und Rheumaklinik Bad Bramstedt

${ }^{2}$ Klinik für Ophthalmologie, Universitätsklinikum Kiel, Christian-Albrechts-Universität Kiel

${ }^{3}$ Klinik für Diagnostische Radiologie, Universitätsklinikum Kiel, Christian-Albrechts-Universität Kiel

Korrespondenz

Dr. med. Peter Lamprecht · Poliklinik für Rheumatologie

Universitätsklinikum Lübeck

und Rheumaklinik Bad Bramstedt · Ratzeburger Allee 160 - 23538 Lübeck · Tel.: 0451/5004798 .

Fax: 0451/5003650 · E-Mail: lamprecht@rheuma-zentrum.de

eingereicht: 7.5 .2002 - akzeptiert: 10.7 .2002 
Die Wegenersche Granulomatose (WG) ist eine Autoimmunerkrankung unbekannter Ätiologie. Organ- und lebensbedrohliche Verläufe gefährden bei voller Krankheitsausprägung den Patienten. Der Tumor-Nekrose-Faktor(TNF)- $\alpha$ spielt bei der Granulombildung und Vaskulitisentstehung eine entscheidende Rolle. Bei der WG werden erhöhte TNF- $\alpha$-Plasmaspiegel und erhöhte Spiegel der löslichen 55 kDa und 75 kDa TNF- $\alpha$-Rezeptoren I und II vorgefunden $(16,18)$. Aktivierte T-Zellen produzieren TNF- $\alpha$ und ein $\mathrm{Th}_{1}$-ähnliches Zytokinprofil $(3,14)$. Hierbei scheint insbesondere der expandierten $\mathrm{CD}^{+} \mathrm{CD}^{+} 8^{-} \mathrm{T}$-Zell-Population eine besondere Rolle zuzukommen. $\mathrm{CD} 4^{+} \mathrm{CD} 28^{-}$T-Zellen zeigen eine restringierte, überwiegende TNF- $\alpha$ - und IFN- $\gamma$-Produktion und sind sowohl im Blut als auch in granulomatösen Entzündungsherden nachzuweisen, wo sie wahrscheinlich aufgrund ihrer Zytokinproduktion zur Rekrutierung weiterer Makrophagen und T-Zellen beitragen $(7,8)$. Eine erhöhte Expression der Chemokin-Rezeptoren CCR5 auf CD28- T-Zellen des peripheren Blutes (9) sowie der Nachweis des Chemokins RANTES (regulated upon activation in normal T-cells, expressed and secreted = CCL5) in granulomatösen Läsionen (2) sind mögliche Hinweise auf eine CCR5-vermittelte Rekrutierung TNF- $\alpha$-produzierender CD28- T-Zellen in die granulomatösen Läsion. TNF- $\alpha$ induziert die Voraktivierung (englisch: priming) und Translokation intrazellulärer Proteinase 3 (PR3 = ,Wegenersches Autoantigen“) auf die Zelloberfläche neutrophiler Granulozyten (4). Antineutrophile zytoplasmatische Antikörper (PR3-ANCA) binden an die PR3 auf der Zelloberfläche, aktivieren die neutrophilen Granulozyten und schädigen das Endothel kleiner Gefäße (19). Die Normalisierung der erhöhten TNF- $\alpha$-Produktion von TZellen und Monozyten scheint eine Vorraussetzung für eine Remission bei der WG zu sein $(10,14)$.

Wir haben kürzlich die über die erfolgreiche Remissionsinduktion mit dem monoklonalen, chimären Anti-TNF- $\alpha$-Antikörper Infliximab bei sechs Patienten, bei denen eine WG auf die Standardtherapie ( $2 \mathrm{mg} / \mathrm{kg} / \mathrm{Tag}$ Cyclophosphamid p.o. und Prednisolon p.o., sog. Fauci-Schema) nicht ansprachen (11). Vor dem Hintergrund teilweise nur kurz anhaltender Remissionen nach einer Therapie mit TNF- $\alpha$-Blockern bei anderen rheumatischen Erkrankungen, z.B. der rheumatoiden Arthritis (21), stellt sich die Frage, wie lange die erzielten Remissionen mit Infliximab bei der WG anhalten? Im Folgenden berichten wir über den Verlauf nach nunmehr 1-2-jähriger Beobachtung.

\section{Patienten und Methodik}

Alle Patienten erfüllten die CHC-Definition (6) und die ACR-Kriterien (13) der WG. Bei drei Patienten bestätigten nasale Biopsien die Diagnose. Bei zwei Patienten wurde bioptisch eine fokal und segmental nekrotisierende Glomerulonephritis nachgewiesen und bei einem Patienten überwiegend granulomatöse Veränderungen in einem Lungenteilresektat. Die Patienten wurden regelmäßig während ihrer stationären Aufenthalte sowie später ambulant in 4-12-wöchigen Abständen evaluiert. Laboruntersuchungen sowie verschieden Untersuchungen zur genauen Erfassung des Organbefalls wie z.B. Röntgen-Aufnahmen des Thorax, hochauflösender Computertomographie (HRCT), bronchoalveoläre Lavage, Kernspintomographie des Kopfes zur Beurteilung des Sinus-, Orbita- und ZNS-Befalls erfolgten gemäß einer
Tab.1 Therapierefraktäre Manifestationen unter einer remissionsinduzierenden Standard-Cyclophosphamid-Therapie $(2 \mathrm{mg} / \mathrm{kg} /$ Tag p.o.) über $\geq 3$ Monate und einem Steroidbedarf von $\geq 20 \mathrm{mg} /$ Tag p.o. bei sechs Patienten mit Wegenerscher Granulomatose. RPGN=Rapid progressive Glomerulonephritis.

\begin{tabular}{|ll}
\hline Patient & Wichtigste refraktäre Manifestation \\
\hline 1 & Drohender Visusverlust durch retroorbitale Granulome \\
\hline 2 & Drohender Visusverlust durch retroorbitale Granulome \\
3 & Drohender Visusverlust durch retroorbitale Granulome \\
\hline 4 & Nierenfunktionseinschränkung bei RPGN, \\
& neue pulmonale Infiltrate \\
\hline 6 & Nierenfunktionseinschränkung bei RPGN \\
\hline
\end{tabular}

standardisierten Untersuchungsfolge und Einbeziehung regelmäßiger konsiliarischer Beurteilungen durch weitere Spezialisten, wie zuvor beschrieben (17). Der Organbefall wurde über den DEI (Disease Extension Index) beschrieben (5), die Vaskulitisaktivität mittels des BVAS (Birmingham Vasculitis Activity Index) erfasst (15). Hierbei wird ein Score ermittelt, der sich aus der Summe des Organbefalls (5) bzw. laborchemisch und klinisch-apparativ erfassbaren, aktiven Vaskulitismanifestationen ergibt (15).

Sechs Patienten mit seit mehr als 3 Monaten bestehender therapierefraktärer WG und einem Steroidbedarf von $\geq 20 \mathrm{mg} / \mathrm{Tag}$ Prednisolon p.o. erhielten Infliximab (Remicade ${ }^{\circledR}$, Essex Pharma, München) additiv zur Standard-Cyclophosphamid-Therapie ( $2 \mathrm{mg} / \mathrm{kg} / \mathrm{Tag}$ ). Patientin 1 und Patient 3 erhielten Infliximab in einer Dosierung von $3 \mathrm{mg} / \mathrm{kg}$ i.v., die übrigen Patienten sowie später die Patienten 6 und 3 (Rezidiv bzw. erneute Therapie zur Remissionsinduktion) in einer Dosierung von $5 \mathrm{mg} / \mathrm{kg}$ i.v., die effektiver erschien (11). Zwischen den ersten beiden InfliximabGaben lag ein 2-wöchiges Intervall. Die weiteren Gaben erfolgten zunächst in 4-wöchigen Intervallen (11) sowie bei der Behandlung des Rezidivs von Patient 6 und bei Patient 3 bei erneuter Remissionsinduktion später auch in 8-wöchigen Abständen (s.u.). Die Behandlung erfolgte bis zur Remissionsinduktion, d.h. dem Fehlen von Zeichen fortbestehender Krankheitsaktivität bei gleichzeitiger Steroiddosis-Reduktion $\leq 7,5 \mathrm{mg} / \mathrm{kg} /$ Tag Prednisolon p.o. („Cushing-Schwelle“) ohne erneute Aktivitätszunahme.

\section{Ergebnisse}

\section{Remissionsinduktion}

Bei sechs Patienten bestand die Krankheitsaktivität unter der Standardtherapie mit Cyclophosphamid ( $2 \mathrm{mg} / \mathrm{kg} / \mathrm{tag}$ p.o.) und Steroiden (> 20mg/Tag p.o. Prednisolon) fort (Tab.1).

Durch die zusätzliche Gabe von Infliximab konnte bei sechs Patienten eine Remission erzielt werden, bei Patient 3 wurde die Therapie mit Infliximab nach der dritten Gabe beendet, weil der Verdacht auf eine systemische Infektion bestand. 


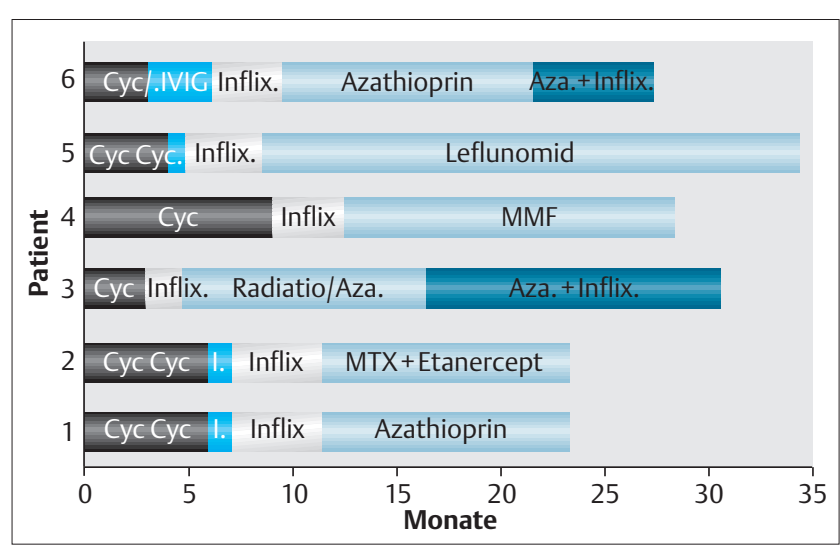

Abb.1 Schematische Darstellung des Therapieverlaufs bei sechs Patienten mit Wegenerscher Granulomatose.

Cyc $=$ Cyclophosphamid p. $0 .(2 \mathrm{mg} / \mathrm{kg})$

Cyc I. = Intensivierte Cyclophsphamid-Therapie $(4 \mathrm{mg} / \mathrm{kg})$,

Inflix. = Infliximab,

Aza. $=$ Azathioprin,

MTX = Methotrexat,

$\mathrm{MMF}=$ Mycophenolat mofetil.

\section{Remissionserhaltung und weitere Verlaufsbeobachtung}

Nach erfolgreicher Remissionsinduktion mit Infliximab konnte die Remission z.T. über 2 Jahre erhalten werden (Abb.1).

Der weitere Verlauf gestaltete sich bei den einzelnen Patienten unterschiedlich:

Patient 1: Bei der 63-jährigen Patientin mit pulmo-renaler Initialmanifestation der WG war es zu mehreren Rezidiven gekommen. Nach 6 Jahren verlor sie den Visus auf dem linken Auge infolge retroorbitaler Granulombildung. Ein erneutes Rezidiv mit pulmonalen Infiltraten, dysmorpher Mikrohämaturie und retroorbitalen Granulomen blieb unter der Standardtherapie mit Cyclophosphamid und Prednisolon sowie unter kurzzeitiger intensivierter Cyclophosphamid-Therapie $(4 \mathrm{mg} / \mathrm{kg} / \mathrm{Tag}$ p.o.) refraktär. Bei drohendem Visusverlust und zwischenzeitlicher Dosislimitierender Cyclophosphamid induzierter Leukopenie konnte eine Remission einschließlich einer Regression der orbitalen Granulombildung durch additive Gabe von sechs Infliximab Infusionen erzielt werden. Zur Remissionserhaltung bekam die Patientin hiernach Azathioprin ( $2 \mathrm{mg} / \mathrm{kg} / \mathrm{Tag}$ p.o.) und weiterhin $5 \mathrm{mg} /$ Tag Prednisolon p.o. Nach 12-monatiger Remission wurde ein niedrig differenzierter, maligner neuro-endokriner Tumor, am ehesten einem atypischen Karzinoid des Bronchus entsprechend, mit Lymphknotenmetastasierung diagnostiziert. Es erfolgten eine Radiatio und Chemotherapie.

Patient 2: Der 53-jährige Patient erlitt unter einer auswärtig durchgeführten, mehrjährigen Cyclophosphamid-Dauertherapie (kumulative Cyclophosphamid Dosis 450 g) erneut ein Rezidiv der WG mit drohendem Visusverlust infolge retroorbitaler Granulombildung. Eine intensivierte Cyclophosphamid-Behandlung über 4 Wochen blieb erfolglos. Nach erfolgreicher Remissionsinduktion mit teilweiser Regression der Orbitagranulome durch sechs Infliximab-Infusionen konnte die Remission über 12 Monate mit Methotrexat und $5 \mathrm{mg} /$ Tag Prednisolon p.o. erhalten werden. Bei hoher kumulativer Cyclophosphamid-Dosis, Rezidivneigung der Orbitagranulome unter alleiniger Cyclophsphamid-Therapie, aber eindeutigem Effekt der TNF- $\alpha$-Blockade, wurde die ambulante Therapie nach der Remissionsinduktion durch die zusätzliche Gabe von Etanercept zu Methotrexat effektiv fortgeführt.

Patient 3: Der 53-jährige Patient war 1999 bei Cyclophosphamid-refraktärer, retroorbitaler Granulombildung im Rahmen eines Rezidivs seiner seit 9 Jahren bestehenden WG durch zusätzliche Infliximab-Gabe behandelt worden. Die Infliximab-Therapie war jedoch nach drei Infusionen infolge einer vermuteten systemischen Infektion bei intermittierendem CRP-Anstieg beendet worden. Der Verdacht einer Infektion bestätigte sich jedoch nicht. Trotz Steroiddosen $\geq 50 \mathrm{mg} /$ Tag Prednisolon p.o. und Radiatio der Orbita konnte in den folgenden 12 Monaten keine dauerhafte Remission erzielt werden. Infolge einer hohen kumulativen Cyclophosphamid-Dosis (275 g) und einer Cyclophosphamid-induzierten, hämorrhagischen Zystitis wurde die Cyclophosphamid-Therapie beendet und der Patient erhielt Azathioprin (2 mg/kg/Tag p.o.). Bei progredienter Protrusio bulbi (Abb.2) und hoher Entzündungsaktivität, kompliziert durch eine tiefe Beinvenenthrombose, erhielt der Patient nunmehr erneut Infliximab additiv zu Azathioprin in einer Dosierung von $5 \mathrm{mg} / \mathrm{kg}$ i.v. mit einem 2-wöchigen Therapie-Intervall zwischen den ersten beiden Infusionen, 4-wöchigen Therapie-Intervallen bei den folgenden vier Infusionen und nachfolgend 8wöchigen Therapie-Intervallen. Nach nunmehr zehn InfliximabInfusionen über einen Zeitraum von 12 Monaten ist eine Remission eingetreten (Abb.3). Die Steroiddosis konnte ohne erneute Aktivitätszunahme der Krankheit von $\geq 50 \mathrm{mg} /$ Tag Prednisolon p.o. auf $5 \mathrm{mg} /$ Tag p.o. reduziert werden. Die Therapie soll mit Azathioprin zur Remissionserhaltung fortgesetzt werden.

Patient 4: Eine anhaltende pulmo-renale Krankheitsaktivität mit führender Nierenfunktionsverschlechterung bei Primärmanifestation der WG konnte bei diesem 64-jährigen Patienten durch vier Infliximab-Infusionen zur Cyclophosphamid-Therapie in Remission gebracht werden. Aufgrund einer allergischen Reaktion auf Azathioprin erhielt der Patient 2 g/Tag Mykophenolat mofetil sowie $5 \mathrm{mg} /$ Tag Prednisolon p.o. zur Remissionserhaltung. Die Remission besteht seit 16 Monaten fort.

Patient 5: Ein renales Rezidiv mit progredienter Nierenfunktionsverschlechterung blieb 1999 unter Standard- und intensivierter Cyclophosphamid-Therapie bei dem 59-jährigen Patienten refraktär. Die Remission wurde durch additive Gabe von vier Infliximab-Infusionen erreicht. Eine Bronchopneumonie wurde antibiotisch behandelt. Nach der Remissions-Induktion blieb die Remission über einen Zeitraum von 26 Monaten unter $20 \mathrm{mg} /$ Tag Leflunomid p.o. und $4 \mathrm{mg} /$ Tag Prednisolon p.o. bis jetzt erhalten.

Patient 6: Die seit 7 Jahren bestehende WG führte bei dem 47jährigen Patienten rezidivierend zu pulmonaler Beteiligung mit kavitierenden, histologisch gesicherten granulomatösen Entzündungsherden. Bei fehlender, dauerhafter Remissionsinduktion durch Cyclophosphamid und intravenöser ImmunglobulinGabe (IVIG) konnte nach 5-jähriger Krankheitsdauer eine Remission durch vier Infliximab-Infusionen erzielt werden. Der 


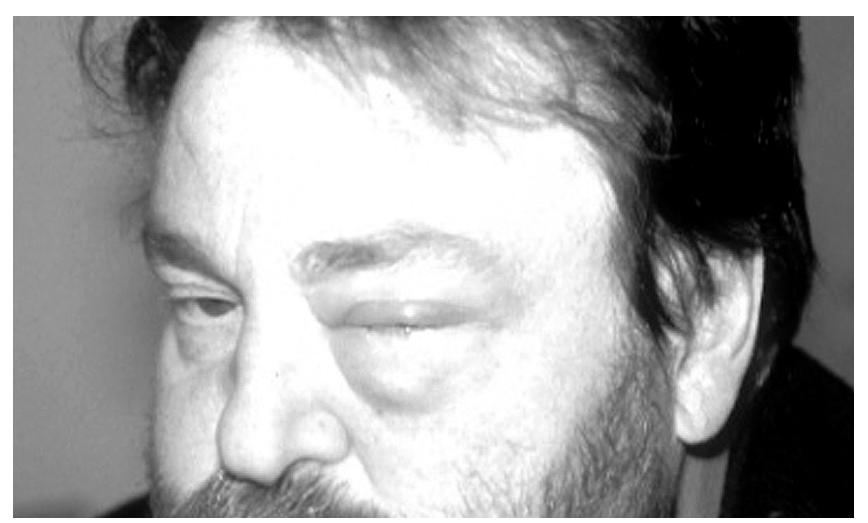

Abb. 2 Bei Patient 3 war die Infliximab-Therapie bei vermuteter systemischer Infektion beendet worden. Bei anhaltender Krankheitsaktivität mit drohendem Visusverlust infolge retroorbitaler Granulome mit Protrusio bulbi und Ineffektivität weiterer therapeutischer Maßnahmen wurde mit Azathioprin in Kombination mit Infliximab behandelt.

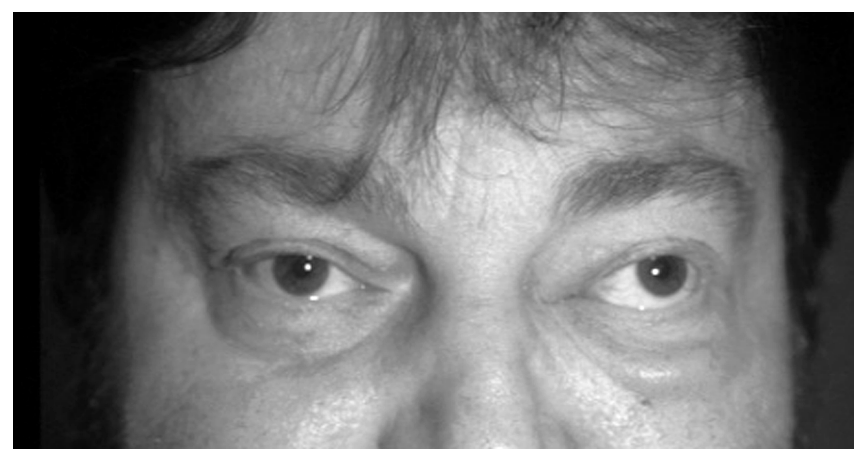

Abb.3 Gleicher Patient wie in Abb.2: Remission nach Behandlung mit Azathioprin in Kombination mit Infliximab über 12 Monate.

Patient blieb in Remission unter Azathioprin ( $2 \mathrm{mg} / \mathrm{kg} / \mathrm{Tag}$ p.o.) und $5 \mathrm{mg} /$ Tag Prednisolon p.o. für 12 Monate. Ein erneutes pulmonales Rezidiv wurde bei hoher kumulativer Cyclophosphamid-Dosis ebenfalls durch eine Therapie-Intensivierung mit additiver Gabe von Infliximab in 8-wöchigem Intervall zur vorbestehenden Azathioprin Therapie überwunden und nach 6 Monaten eine Remission erzielt, so dass eine erneute fortgesetzte Remissionserhaltung mit Azathioprin in höherer Dosierung oder mit Methotrexat geplant ist. Die erneute Remissionsinduktion war einmalig durch eine akute, eitrige Bronchitis kompliziert worden, die sich unter antibiotischer Therapie besserte.

Während die Infliximab-induzierte Remissionsinduktion bei allen Patienten zur Reduzierung des DEI (Disease Extension Index) und der BVAS-Werte (Birmingham Vasculitis Activity Score) auf 0 sowie der C-ANCA Titer auf 0 geführt hatte (11), waren der BVAS.1-Score und der DEI beim Rezidiv des Patienten 6 erneut mit 9 bzw. 3 erhöht. Der C-ANCA-Titer und PR3-ANCA war bei diesem Patienten zu keinem Zeitpunkt nachweisbar (ANCA-negative WG), so dass auch das Rezidiv nicht zu einer entsprechenden Titer-Bewegung führte.

\section{Diskussion}

Wir berichten über die weitere Beobachtung von sechs Patienten, bei denen bei zuvor therapierefraktärem Verlauf unter der Standardtherapie mit Cyclophosphamid ( $2 \mathrm{mg} / \mathrm{kg} / \mathrm{Tag}$ p.o.) und Prednisolon gemäß dem Fauci-Schema eine Remission erfolgreich durch die additive Gabe von Infliximab induziert wurde (11). Bei allen sechs Patienten wurde nach 4-6 Infusionen eine Remission erzielt. Bei einem wurde Patienten die Infliximab Therapie wegen einer vermuteten systemischen Infektion beendet (11). Bei Ineffektivität anderer Therapiemaßnahmen wurde dann jedoch erneut eine Infliximab Therapie, diesmal in der von uns als effektiver betrachteten höheren Dosis von $5 \mathrm{mg} / \mathrm{kg}$ i.v., begonnen und führte zur Remissionsinduktion. Bei vier Patienten waren andere Therapieoptionen, die bei therapierefraktärem Verlauf propagiert wurden, d.h. die risikobelastete intensivierte Cyclophosphamid-Therapie ( $4 \mathrm{mg} / \mathrm{kg} / \mathrm{Tag}$ p.o.) und IVIG, zuvor ineffektiv (11). Wie in der vorgelegten Beobachtung dargestellt, sind vier Patienten nach 16-26 Monaten weiterhin in Vollremission. Remissionen können also mit Infliximab bei therapierefraktärer WG induziert und anschließend mit einer remissionserhaltenden Therapie aufrecht erhalten werden.

Bei einer Patientin wurde 12 Monate nach der erzielten Remission ein Bronchus-Karzinoid diagnostiziert. Ein Zusammenhang mit der Therapie oder der Grunderkrankung ist derzeit nicht direkt zu beweisen. Jedoch wurde beispielsweise für alkylierenden Substanzen, insbesondere hoher kumulativer Cyclophosphamid-Dosis (>100 g), wiederholt ein erhöhtes Risiko für bestimmte maligne Tumoren, z.B. Blasentumoren und myelodysplastische Syndrome nach zum Teil langjähriger Latenz, bei Patienten mit WG berichtet (17).

Ein Patient erlitt unter der remissionserhaltenden Therapie mit Azathioprin und niedrig dosierter Steroid-Gabe nach 12 Monaten ein Rezidiv. Bei beiden Patienten konnte bei pulmonalem Rezidiv bzw. weiterhin progredienter, visusbedrohender Protrusio bulbi, hoher kumulativer Cyclophosphamid-Dosis (100 g bzw. 275 g) und Cyclophosphamid-induzierter hämorrhagischer Zystitis durch erneute Gabe von Infliximab zusätzlich zu Azathioprin eine Remission erzielt werden. Eine ex vivo Analyse der TNF- $\alpha-$ Produktion von T-Zellen und Monozyten bestätigt, dass eine Normalisierung der TNF- $\alpha$-Produktion eine Vorraussetzung für eine Remission der WG zu sein scheint, wohingegen eine ineffektive Therapie wahrscheinlich die TNF- $\alpha$-Produktion von T-Zellen und Monozyten nicht ausreichend kontrolliert $(10,14)$. Der additive Therapieeffekt von Infliximab sowohl bei ineffektiver Cyclophosphamid- wie auch Azathioprin-Therapie kann hierdurch erklärt werden. Die TNF- $\alpha$-Blockade gewinnt auch in der Therapie anderer primär systemischer Vaskulitiden, bei denen TNF- $\alpha$ eine Rolle spielt, an Bedeutung. So konnten wir kürzlich bei drei Patienten mit therapierefraktärem Verlauf eines Churg-Strauss-Syndrom eine Remission durch additive Gabe von Infliximab zur vorbestehenden Therapie induzieren (1).

Neben dem monoklonalen, chimären Anti-TNF- $\alpha$-Antikörper Infliximab, der sowohl mit dem löslichen TNF- $\alpha$-Homotrimer als auch mit der membrangebundenen Form interagiert und zur Lyse TNF- $\alpha$-exprimierender Zellen führt (21), steht mit dem löslichen, an eine humane Fc-Region gekoppelten 75 kDa TNF- $\alpha$ - 
Rezeptor-Dimer Etanercept derzeit eine weitere TNF- $\alpha$-blockierende Substanz zur Therapie zur Verfügung. Etanercept bindet neben löslichem TNF- $\alpha$ auch Lymphotoxin- $\alpha$ (21). Etanercept ist in zwei Phase-I/II-Studien bei nicht lebensbedrohlichen WGManifestationen und anhaltender bzw. neu aufflammender Krankheitsaktivität bei jeweils 20 Patienten zusätzlich zu MTX oder einer anderen vorbestehenden Therapie gegeben worden $(12,20)$. In beiden Studien wurden Remissionen erzielt und der BVAS vermindert. Eine intermittierende Krankheitsaktivität bei mehreren Patienten und eine progrediente retroorbitale Beteiligung bei einem Patienten wurde in einer der beiden Studien berichtet (20). Eine erhöhte Infektionsrate insbesondere respiratorischer Infektionen wurde ebenfalls berichtet (20).

In unserer Studie der sechs Patienten waren eine vermutete, aber nicht bestätigte, systemische Infektion, eine Bronchopneumonie und eine akute, eitrige Bronchitis unter der zusätzlichen Gabe von Infliximab beobachtet worden. Vor dem Hintergrund einzelner Berichte über Tuberkulose-Reaktivierungen unter der Therapie mit TNF- $\alpha$-Blockern (21) ergingen kürzlich seitens des Paul Ehrlich-Instituts, Bundesamt für Sera und Impfstoffe, Empfehlungen zur Diagnose und Prävention einer latenten Tuberkulose vor Beginn einer Behandlung mit Infliximab. Die Diskussion über Nebenwirkungen der Infliximab-Therapie muss allerdings vor dem Hintergrund therapieassoziierter Komplikationen bei anderen Immunsuppressiva gesehen werden. Zudem ist die bisher schlechte Prognose therapierefraktärer Verläufe der WG, die ca. 5-10\% der Patienten betreffen (17), in Rechnung zu stellen.

\section{Fazit}

Zusammenfassend lässt sich feststellen, dass die TNF- $\alpha$-Blockade die therapeutischen Optionen bei therapierefraktärem Verlauf einer WG erweitert hat und somit bei diesen Patienten eine günstigere Prognose als bisher besteht. Remissionen von 16-26 Monaten wurden bis jetzt erzielt. Unter den Nebenwirkungen sind insbesondere die Infektgefährdung zu nennen. In weitere Studien müssen die Möglichkeiten und die Stellung der TNF- $\alpha$-Blockade in der Therapie der WG bestimmt werden.

Autorenerklärung: Die Autoren erklären, dass sie keine finanziellen Verbindungen mit einer Firma haben, deren Produkt in dem Artikel erwähnt wird, oder mit einer Firma, die ein Konkurrenzprodukt vertreibt.

\section{Literatur}

1 Arbach O, Lamprecht P, Nölle B, Heller M, Gross WL, Gause A. Three case reports: treatment of refractory Churg-Strauss syndrome (CSS) by TNF-blockade (Abstract-Band I). Med Klinik 2002; 97: 155

2 Coulomb-L'Hermine A, Capron F, Zou W et al. Expression of the chemokine RANTES in pulmonary Wegener's granulomatosis. Hum Pathol 2001; 32: 320-326

3 Csernok E, Trabandt A, Müller A et al. Cytokine profiles in Wegener's granulomatosis: predominance of type 1 (Th1) in the granulomatous inflammation. Arthritis Rheum 1999; 42: 742-750

${ }^{4}$ Csernok E, Ernst M, Schmitt WH, Bainton DF, Gross WL. Activated neutrophils express proteinase 3 on their plasma membrane in vitro and in vivo. Clin Exp Immunol 1994; 95: 244-250

${ }^{5}$ DeGroot K, Gross WL, Herlyn K, Reinhold-Keller E. Development and validation of a disease extent index for Wegener's granulomatosis. Clin Nephrol 2001; 55: 31-38

${ }^{6}$ Jennette JC, Falk RJ, Andrassy K et al. Nomenclature of systemic vasculitides. Proposal of an international consensus conference. Arthritis Rheum 1994; 37: 187-192

7 Komocsi A, Lamprecht P, Csernok E et al. Peripheral blood and granuloma CD4+CD28- T-cells are a major source of IFN- $\gamma$ and TNF- $\alpha$ in Wegerner's granulomatosis. Am J Path 2002; 160: 1717-1724

8 Lamprecht P, Moosig F, Csernok E et al. CD28 negative T cells are enriched in granulomatous lesions of the respiratory tract in Wegener's granulomatosis. Thorax 2001; 56: 751-757

9 Lamprecht P, Erdmann A, Müller A, Csernok E, Gross WL. Chemokine receptor expression on CD4-positive CD45RO-positive memory Tcells in Wegener's granulomatosis (abstract). Arthritis Rheum 2001; 44 (Suppl): S391

${ }^{10}$ Lamprecht P, Kumanovics G, Müller A et al. Elevated monocytic IL-12 and TNF- $\alpha$ production in Wegener's granulomatosis is normalized by cyclophosphamide and corticosteroid therapy. Clin Exp Immunol 2002; 128: 181-186

${ }^{11}$ Lamprecht P, Voswinkel J, Lilienthal T et al. Effectiveness of TNF- $\alpha$ blockade with infliximab in refractory Wegener's granulomatosis. Rheumatology 2002; in Druck

12 Langford CA, Talar-Williams C, Barron KS, McCabe KE, Sneller MC. Phase I/II trial of etanercept in Wegener's granulomatosis (WG): safety and preliminary experience (abstract). Arthritis Rheum 2000; 43 ( Suppl): S163

${ }^{13}$ Leavitt RY, Fauci AS, Bloch DA et al. The American College of Rheumatology 1990 criteria for the classification of Wegener's granulomatosis. Arthritis Rheum 1990; 33: 1101-1107

${ }^{14}$ Ludviksson BR, Sneller MC, Chua KS et al. Active Wegener's granulomatosis is associated with HLA-DR+ CD4+ T cells exhibiting an unbalanced Th1-type T cell cytokine pattern: reversal with IL-10. J Immunol 1998; 160: 3602-3609

15 Luqmani RA, Bacon PA, Moots RJ et al. Birmingham Vasculitis Activity Score (BVAS) in systemic necrotizing vasculitis. Q J Med 1994; 87: 671-678

${ }^{16}$ Noronha IL, Kruger C, Andrassy K, Ritz E, Waldherr R. In situ production of TNF- $\alpha$, IL- $1 \beta$ and IL-2R in ANCA-positive glomerulonephritis. Kidney Int 1993; 43: 682-689

${ }^{17}$ Reinhold-Keller E, Beuge N, Latza U et al. An interdisciplinary approach to the care of patients with Wegener's granulomatosis. Longterm outcome in 155 patients. Arthritis Rheum 2000; 43: 1021-1032

18 Roux-Lombard P, Lin HC, Peter JB, Dayer JM. Elevated serum levels of TNF soluble receptors in patients with positive anti-neutrophil cytoplasmic antibodies. Br J Rheumatol 1994; 33: 428-431

19 Savage COS, Harper L, Holland M. New findings in pathogenesis of antineutrophil cytoplasm antibody-associated vasculitis. Curr Opin Rheumatol 2002; 14: 15-22

20 Stone JH, Uhlfelder M, Hellmann D, Crook S, Bedocs NM, Hoffman GS. Etanercept combined with conventional treatment in Wegener's granulomatosis: a six-months open-label trial to evaluate safety. Arthritis Rheum 2001; 44: 1149-1154

21 Taylor PC. Anti-tumor necrosis factor therapies. Curr Opin Rheumatol 2001; 13: 164-169 